\title{
Borderline Personality Pathology, Polysomnography, and Self-Reported Sleep Problems: A Review
}

\author{
Joshua R. Oltmanns ${ }^{1}$ - Thomas F. Oltmanns ${ }^{2}$
}

Published online: 21 April 2015

(C) Springer International Publishing AG 2015

\begin{abstract}
There is a growing body of research that links borderline personality pathology to sleep disturbance through polysomnography (PSG) and self-report studies. Twelve PSG studies are reviewed that found sleep differences in recordings of sleep parameters such as sleep continuity, nonREM sleep, and REM sleep in borderline personality disorder (BPD) patients compared to controls. Further, since the turn of the century, self-report methodology has been increasingly utilized to investigate this relationship, and findings from these studies are reviewed. The evidence suggests that borderline personality pathology is uniquely associated with sleep disturbance. Future directions for this research are discussed.
\end{abstract}

Keywords Borderline personality disorder · Personality pathology · Sleep problems · Insomnia · Polysomnography · Personality $\cdot$ Review

\section{Introduction}

Borderline personality disorder (BPD) is defined in terms of a collection of maladaptive personality traits associated with extreme emotional instability, self-control dysregulation, and

This article is part of the Topical Collection on Sleep and Psychological Disorders

Joshua R. Oltmanns

jroltmanns@uky.edu

Thomas F. Oltmanns

toltmann@wustl.edu

1 Department of Psychology, University of Kentucky, 111-D Kastle Hall, Lexington, KY 40506-0044, USA

2 Washington University in St. Louis, Campus Box 1125, One Brookings Drive, St. Louis, MO 63130-4899, USA interpersonal problems [1]. These maladaptive personality traits are relatively stable across the lifespan, occur in about $2.9 \%$ of the population [2], and interfere with social, occupational, cognitive, and relational functioning [3-5]. Individuals with BPD focus on negative stimuli and have more pessimistic beliefs about themselves and about the world [3]. They show severe levels of social dysfunction [5], and BPD is highly comorbid with substance use disorders [6]. People suffering from borderline personality pathology are at greater risk for suicide, mood disorders, anxiety disorders, and health problems [7-10].

In addition to health problems such as cardiovascular disease, obesity, and arthritis, there is a growing body of research suggesting a link between borderline personality pathology and sleep problems (e.g., $[11 \bullet, 12 \bullet])$. Sleep problems associated with insomnia lead, in turn, to impairment in areas such as physical, psychological, and occupational functioning [13]. Sleep seems to play a central role in mood regulation [14], which occupies a central position in the phenomenology of BPD [15]. Thus, the examination of sleep difficulties associated with borderline personality pathology is an important, and potentially highly beneficial, route of investigation.

The association between borderline personality pathology and sleep problems has been investigated using multiple methods to assess both personality and sleep. Studies using polysomnography (PSG) have found differences between borderline patients and controls that are similar to the differences between patients with major depressive disorder (MDD) and controls. There are discrepancies among findings in these studies (possibly due to inconsistent methods of BPD assessment, small sample sizes, varying gender distributions, and different clinical characteristics of the studies), yet the results demonstrate convincingly that abnormalities in PSG recordings of sleep continuity, non-REM sleep, and REM sleep are common in patients with BPD. The relation between 
borderline personality pathology and sleep problems has also been investigated using self-report methodology. These studies, which have emerged more recently, have corroborated the presence of sleep problems in borderline pathology. In the following pages, we will review PSG studies as well as those based on self-report methods.

\section{Polysomnographic Studies}

PSG studies that examined the sleep of borderline patients started being published soon after BPD was introduced to the official diagnostic manual in 1980 [16]. Investigators had already taken notice of the high diagnostic comorbidity of BPD with MDD [17]. Initially, it was debated whether observations of shortened REM latency in BPD patients should be taken as evidence that the disorder was best classified as a mood disorder rather than a personality disorder [18-21, 54]. Shortened REM latency is often found in MDD [22], and it has been argued that this finding suggests a common biological etiology for BPD and mood disorders (e.g., $[18,23,24])$. However, with the increased research interest in BPD over the years, and discrepant findings in the literature on BPD and sleep, this research has become focused on understanding what it is about BPD that might cause sleep disturbance, rather than what this relationship can tell us about how the disorder should be classified.

Twelve studies that compared PSG sleep characteristics of BPD patients to those of various control groups were reviewed for the present article. The 12 studies consisted of younger adult ( $M$ ages in the 20 s and 30 s) and mostly female BPD samples whose sleep recordings were compared with those of healthy control groups. Some controlled strictly for comorbid MDD (i.e., excluded patients with any lifetime history of MDD), some less strictly (no current MDD), and some not at all (patients could meet diagnostic criteria for comorbid MDD). Clinical assessment methodology was good across the 12 studies. The majority assessed BPD according to DSM, Research Diagnostic, Feighner, or ICD criteria using semistructured interviews, with the exception of one study that relied on a symptom checklist [23] and one that did not specify how the criteria were assessed [25]. Battaglia et al. [19, 54] included an additional self-report measure (which was used to cross-validate interview diagnosis), three studies included two semi-structured interviews assessing BPD [26•, 27, 28], and one study relied on a consensus diagnosis by two separate interviewers [20]. Five of the studies assessed only inpatients being treated for BPD [19, 20, 25, 27, 28, 54]; one included eight inpatients and two outpatients [21], three consisted of all outpatients $[18,23,26 \cdot]$, one consisted of all inmates in a psychiatric facility accused of violent crimes [29], and two did not specify this information $[12 \bullet, 30]$. The studies were conducted in the USA and Europe, with one in Egypt [23]. All but four studies $[18,20,28,29]$ specified that patients had not taken psychotropic medication for at least 2 weeks prior to study time. All included at least two nights of PSG recording (except Asaad et al. [23], which only had one in some cases), with six including one adaptation night from which data was not used in statistical analyses. A table with all of this information can be requested from the first author.

\section{Polysomnographic Findings}

Important sample characteristics and PSG sleep findings in BPD patients compared with healthy controls are displayed in Table 1. Inspection of this table indicates that, across all studies and sleep parameters, a robust consensus has not emerged. Conclusions can be drawn based on some repeated findings, however, and future research may build upon what has already been demonstrated.

Regarding sleep continuity, significant differences have been found in BPD patients compared to controls. These include: less total sleep time (TST), decreased sleep efficiency, longer sleep onset latency (SOL), and more frequent arousals/wake after sleep onset (WASO) (Table 1). Less total sleep time and sleep efficiency is the slightly more consistent finding, while increased sleep onset latency and increased arousals/WASO are split almost evenly between significant and non-significant differences compared to controls. Gender does not seem to moderate these findings with the exception of SOL, which was only found with all/ majority of female samples. These differences were also found in samples with and without comorbid MDD. Thus, it seems that although there are nearly even splits in two of the four sleep continuity parameters, sleep continuity appears to be significantly impaired in patients with BPD regardless of gender or comorbid MDD.

Regarding non-rapid eye movement (NREM) sleep, differences have been reported in stage I (increased), stage II (decreased), stage III (decreased), and stage IV (both decreased and increased) sleep in BPD patients compared to controls. With the exception of observed differences in stage IV/slow wave sleep (SWS), which are nearly unanimous, studies finding differences in other stages of BPD patients' NREM sleep are in the minority. The finding of both increased and decreased stage IV sleep in BPD may indicate an extreme variability rather than an irregular amount in either direction. However, modern scoring criteria [31] have combined stage III and IV due to an absence of scientific evidence to support a delineation between these sleep stages. When SWS has been investigated in BPD groups as a combination of both stage III and stage IV sleep, only two out of seven studies have found significantly different SWS percentages in BPD groups compared to control groups. 


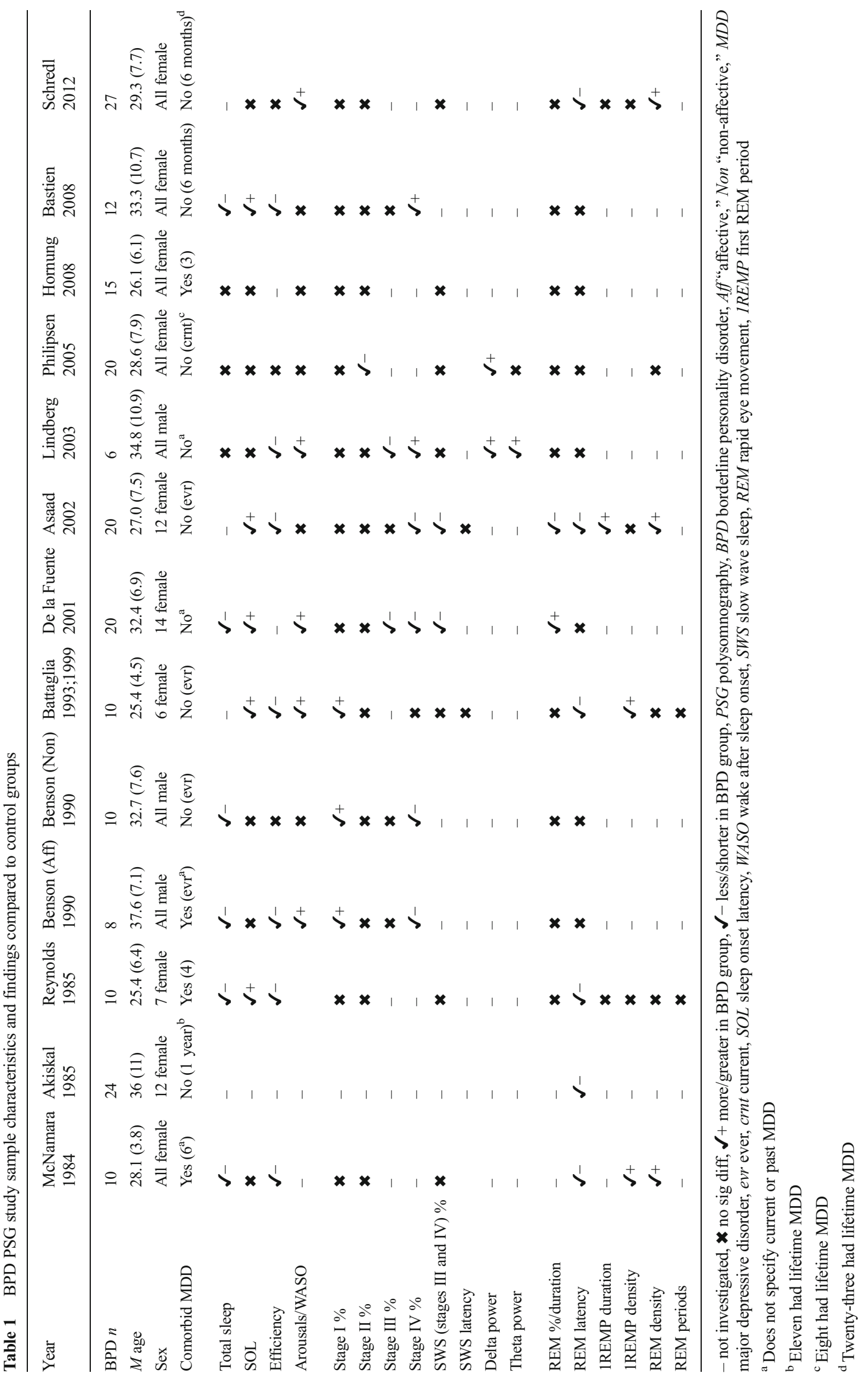


Significantly increased delta power has been reported in both of two studies that have investigated it in BPD compared to controls $[29,30]$. This pattern was found in one all-male study and one all-female study, indicating that this finding is not moderated by gender. Lindberg et al. [29] also found increased theta power, and although it was not statistically significant in the Philipsen et al. [30] study, they also found a trend towards a statistically significant increase in theta power. The finding of increased delta and theta power in the sleep of BPD patients may be indicative of improved in-study sleep [30]. Additional studies investigating spectral power of sleep in BPD patients are warranted.

Regarding REM sleep, significant differences have been found between BPD patients and controls in REM duration (increased and decreased), density (increased), and latency (decreased). Initially, investigators focused on the finding of shortened REM latency in BPD, specifically, because shortened REM latency is characteristic of MDD and other mood disorders (e.g., [22]). Some argued that BPD is better conceptualized as a mood disorder because of this finding (e.g., [18] and later [27]). However, after three initial studies shared this finding, most subsequent studies have not found shortened REM latency in BPD groups relative to controls. Fewer studies have focused on differences between BPD patients and controls specifically with regard to the first REM period of sleep, but significant differences have been found: Longer first REM period duration was found in one study (out of three investigating it), and increased REM density in the first REM period was found in two studies (out of five investigating it) (see Table 1). Overall nightly REM density has been investigated in six studies, and a significant increase was found in BPD patients in half of these studies. Based on inspection of Table 1, comorbid MDD did not seem to moderate findings involving REM density.

These findings from PSG studies suggest that BPD has a unique association with sleep disturbance beyond the influence of its comorbidity with MDD (e.g., [19, 54]). It should be emphasized, however, that these differences have not been found in all studies. Inconsistencies can be found in Table 1 . Some of these inconsistencies may be attributed to the presence or absence of comorbid depression, which influences the relationship between BPD and sleep patterns [17, 20]. Failures to replicate findings in this area may also be the product of varying levels and combinations of symptoms used to define BPD, which is clearly a heterogeneous diagnostic construct (any five of nine DSM criteria can be present to diagnose BPD). Nevertheless, studies in which sleep has been measured objectively have confirmed the presence of sleep irregularities in BPD. Sleep disturbance in BPD is similar to that associated with MDD, but it also appears independent of MDD.

\section{Self-report Studies}

PSG studies of BPD and sleep began to include self-report measures of sleep quality in the 2000s. These measures demonstrated that complaints of sleep problems are characteristic of borderline patients. Since then, studies of the relationship between BPD and sleep problems have become more frequent, and samples in these studies have gotten larger. Selfreport enables quicker, less expensive assessments of sleep disturbance, and with larger sample sizes, statistical control can be used to investigate relationships between BPD, sleep, and comorbid psychopathology. Self-report methods offer some advantages and provide important opportunities to address questions about BPD and sleep quality that could not be answered using PSG methods.

Beginning with a study conducted by Assad and colleagues [23], self-report measures of sleep quality were increasingly used in conjunction with PSG recordings in studies of BPD and sleep. They found that $45 \%$ of the BPD patients selfreported sleep problems, in comparison to only $10 \%$ of controls. Philipsen et al. [30] and Hornung et al. [25] both found that BPD subjects complained of greater sleep difficulty in the month prior to their studies than controls on the Pittsburgh Sleep Quality Index (PSQI [32]). Schredl et al. [12•] also found significantly impaired self-reported sleep quality in a BPD group compared to controls. Following the addition of self-report measures into PSG studies, further studies utilizing solely self-report have become more frequent. These studies are able to obtain data from larger numbers of participants, increasing generalizability of the results. A summary of findings from recent studies of BPD and sleep utilizing self-report can be found in Table 2 .

Semiz and colleagues [33] were the first to examine BPD and sleep without PSG recordings in their study of nightmare disorder, dream content, and sleep quality in a large clinical sample of BPD patients. They reported that $96 \%$ of their group of 88 (48 female, 40 male) nondepressed BPD patients self-identified as poor sleepers, as compared to $12 \%$ of controls. They also found that BPD patients had a significantly higher rate of nightmares and dream anxiety than controls. Nightmares have been found to be significantly more prevalent in BPD groups than in controls in multiple studies [12•, 33, 34].

Harty and colleagues [35] found that borderline pathology was significantly correlated with a subscale of sleep problems on the Personality Assessment Inventory (PAI [36]) in an even larger sample of 513 jail inmates. Partial correlations between borderline pathology and sleep disturbance remained significant even after statistically controlling for depressive symptoms and substance dependence. Of course, exclusive reliance on participants in a correctional facility raises serious questions about the generalizability of these findings, both in terms of the BPD features 


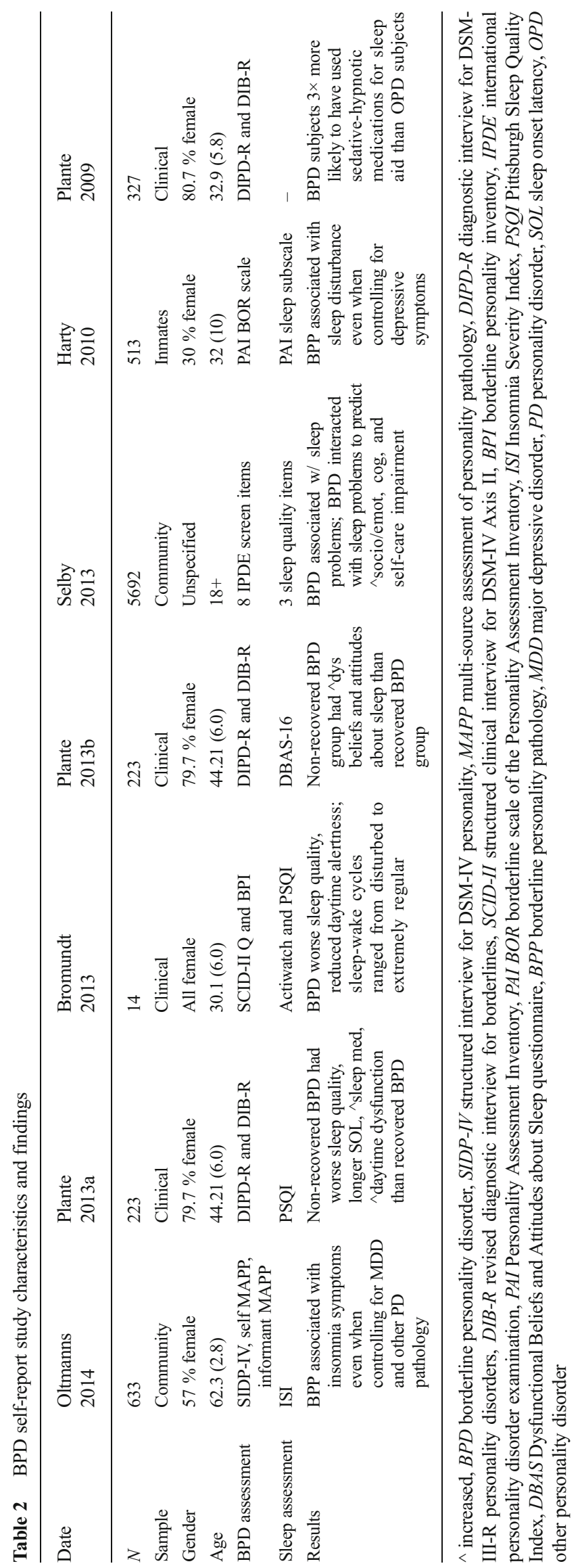

that would be observed as well as the number and quality of sleep problems that might be experienced in a coercive and probably crowded environment.

Plante and colleagues conducted a number of studies regarding BPD and sleep using data from the McLean Study of Adult Development (MSAD [37]), a longitudinal study of a large sample of people who have been treated for borderline and other personality disorders. First, they showed that over the course of multiple 2-year follow-ups, BPD patients were three times more likely to have used sedative-hypnotic medications for sleep aid than patients with other personality disorders [38]. With the same longitudinal sample, Plante, Frankenburg, Fitzmaurice, and Zanarini [39] found that nonrecovered BPD patients (i.e., a group that was diagnosed with BPD at the beginning of the study, and still qualified for the diagnosis 16 years later) had significantly more sleep continuity and daytime dysfunction problems compared to recovered BPD patients (i.e., people who had been diagnosed with BPD in the past, but no longer met diagnostic criteria and had good concurrent psychosocial functioning). In another study, they found that the BPD group held significantly more dysfunctional beliefs and attitudes about sleep than the recovered group [40]. These observations supplement prior research documenting subjective sleep difficulties in BPD and provide unique information suggesting that people with borderline pathology not only suffer from difficulties with sleep but also use more sleep medications to alleviate these problems and are more likely to have dysfunctional beliefs about sleep. These studies are important for a variety of reasons, including the fact that the data are from a large, well-respected investigation of carefully diagnosed patients who have been studied for several years [37]. Rich data from this study may eventually allow for even more detailed consideration of the role that BPD and sleep problems play in relation to other substantial health problems as these middle-aged patients approach later life.

\section{BPD and Sleep in Older Adults and in the Community}

The research that we have reviewed thus far suggests strongly that there is a connection between borderline personality pathology and sleep problems in younger adults, particularly in clinical samples. More recent evidence has extended these findings in large community samples and has expanded the age range in which the effect is observed. Using data from the National Comorbidity Survey Replication (NCS-R [41]), Selby [42] reported that symptoms of BPD were significantly related to self-reported sleep disturbance in a nationally representative, community-based sample of 5692 participants aged 18 and up. Data reported in this paper represent a significant advance beyond earlier studies because of the large, carefully 
ascertained sample from which they were collected. However, borderline pathology was assessed using only a brief, selfreport screening questionnaire, leaving some doubt about the validity of the diagnostic information. Furthermore, because the data were not analyzed with regard to age, it is not clear whether the connection between sleep problems and symptoms of BPD fluctuates or remains relatively stable across the lifespan.

Oltmanns et al. [11•] examined a representative community sample of older adults in the St. Louis area (St. Louis Personality and Aging Network, SPAN [43]; $M_{\text {age }}=62.3$ years) who were assessed with multiple methods for borderline personality pathology (interview, self-report, and informant-report). This method provided a robust assessment of personality pathology and protected against the possible influence of method variance (i.e., minimized the possibility that sleep problems appear to be related to symptoms of BPD because both were measured with self-report instruments that merely reflect a willingness to describe one's problems). We found that borderline personality pathology was significantly associated with sleep problems after controlling for demographic variables, MDD, and all other forms of personality pathology. This finding suggests not only that borderline personality pathology is uniquely predictive of sleep problems in younger adults, but also that this effect can be observed across the lifespan. It holds in community samples where levels of borderline pathology are less marked as well as in clinical samples of younger patients who meet (or have met) full diagnostic criteria for BPD.

\section{Sleep Context and the Validity of Self-reported Sleep Problems}

Philipsen et al. [30] and Hornung et al. [25] both found that BPD patients, in comparison to controls, complained of significantly more total sleep difficulties on the PSQI in the month prior to the PSG study. Philipsen et al. noted that these complaints did not align with their PSG sleep parameter findings, and then suggested that this discrepancy might reflect altered sleep perception in BPD. Interestingly, in both studies, three areas (SOL, TST, and WASO) were measured via self-report specifically for the PSG recording nights as well. The BPD patients did not report impairment in these specific areas for the nights of recording in either study $[25,30]$. The objective measures did not find discrepancies in these areas either. In other words, for SOL, TST, and WASO, BPD patients said in the lab via self-report that they were no different from controls on these parameters, and when measured objectively, they were no different from controls on those parameters. This finding, which was reported in both studies, actually suggests an accurate perception of sleep in BPD. Consideration of this issue brings our attention to another related and interesting issue, which might be called sleep context; inconsistencies between sleep experiences in the lab and sleep experiences at home prior to the lab sessions may lie at the heart of these complex patterns of findings.

Bastien et al. [26•] has offered the interesting hypothesis that BPD patients may sleep better during PSG study recording nights because of the nature of their personality pathology. People with borderline pathology are often lonely and crave companionship. Indeed, interpersonal dysfunctions - fear of abandonment and sensitivity to rejection-may represent the core features of this disorder [44]. During study nights, patients with BPD may sometimes sleep better than they do at home because they feel socially connected (i.e., to the researchers, to the laboratory, and so on). The finding of increased delta and theta power in the sleep of BPD patients during study nights may support this idea [29, 30]. The sleep difficulties reported on the PSQI by BPD patients in the Philipsen et al. [30] and Hornung et al. [25] studies referred to the previous 1 month, when the patients were not in the lab, possibly felt less connected, and were struggling with their symptoms on their own. Based on the accuracy of their perceptions of sleep in the lab during the study nights, it is probable that the self-reported sleep difficulty on the PSQI for the preceding month is also accurate.

Bastien and colleagues [26.] found that BPD patients reported worse total Insomnia Severity Index scores (ISI [45]), less sleep efficiency, less TST, and more total wake time than a group of controls. The ISI scores of the BPD group were similar to those of two insomnia groups. Bastien et al. reported that among four groups (BPD, two insomnia groups, and one group of controls), the accuracy of the BPD group between subjective and objective measures of sleep continuity was the strongest of the groups across the three nights. Schredl et al. [12•] also found congruence between self-report estimates of BPD patients' sleep quality and their PSG sleep quality, but the self-reports in that study were regarding the prior 2 weeks. These findings suggest that during study nights, BPD patients are able to self-report sleep quality with validity, sometimes even more so than the insomnia and control groups. They also illustrate how the inclusion of self-report measures of sleep quality in tandem with PSG recording can address questions that have gone previously unanswered, and even unasked, in the past.

In summary, these findings suggest that perhaps there is not actually altered perception of sleep specific to BPD. Rather, when BPD patients are in the sleep laboratory, they may experience an improvement in their sleep. Studies agree that there are both self-reported and PSG findings of sleep disturbance in borderline pathology. It is possible that BPD patients sleep better during study nights, however, and are able to report this accurately. 


\section{Conclusions}

Several factors may account for the complex and somewhat inconsistent PSG findings regarding specific sleep parameters in BPD. One possible explanation is that discrepancies reflect fundamental classification and diagnostic issues that have recently been the subject of intense debate in the personality disorder field [46, 47]. Categorical diagnoses of PD according to the DSM have come under fire for not fully covering personality pathology, including an arbitrary diagnostic boundary, and being overly heterogeneous [48]. With a heterogeneous diagnostic system of classification for BPD, it makes sense that sleep correlates of the disorder would be heterogeneous as well.

Borderline pathology can be understood in terms of normal personality traits from the five-factor model (FFM) of personality [49]. Within each of the five domains, there are six lowerorder facets that describe personality more specifically. Studies of more specific personality traits and health-related problems have produced important findings for many years [50]. For example, Smith, Glazer, Ruiz, and Gallo [51] found that hostility, anger, and aggressiveness are related to cardiovascular problems. Turiano, Spiro, and Mroczek [52] found that the facet of creativity is associated with better health in men. That literature can be used as a template to expand and enrich research aimed at understanding sleep problems and BPD. Borderline personality pathology has been translated into maladaptive variants of normal personality traits [53]. A more nuanced investigation into maladaptive, borderline personality traits and sleep problems could be provided by a focus on specific personality facets in research. Findings using this system of classification for borderline pathology could help to make findings of the relationship between BPD and sleep more specific and organized, thereby helping us better understand which traits and personality characteristics drive this relationship.

Another explanation for the discrepant findings in this literature is that MDD is so highly comorbid with borderline personality pathology. Studies attempting to exclude MDD in prior research have been useful in helping researchers tease apart the relationship between BPD and sleep disturbance, but comorbid depression and depressed mood are seemingly intrinsic to borderline pathology. Excluding links to depression fully would leave researchers with highly selective and atypical samples of people with borderline pathology. Battaglia et al. [19, 54] excluded a full $47 \%$ of BPD patients being screened for their study in an attempt to have a "clean" sample of BPD. In fact, given the chronic problems with emotion regulation that are characteristic of $\mathrm{BPD}$, it seems reasonable to expect that depression is necessarily an inherent part of this diagnostic construct. Attention should be paid to comorbidities in samples of BPD patients, yet as BPD is highly comorbid with many different forms of psychopathology, this will take considerable effort. Nevertheless, we do currently have enough information to conclude that tangible sleep disruptions are frequently associated with BPD. Any specific finding that may be of particular interest, though, for example shortened REM latency, will need further study using PSG and selfreport methodology in tandem to eliminate moderating factors.

The examination of the relationship between borderline pathology and sleep disturbance has gained increased attention over the past decade. Findings have been almost unanimous that BPD patients and people with borderline pathology selfreport sleep difficulties and display more PSG sleep abnormalities than controls. Self-report studies of BPD and sleep have added value to information about this relationship by enabling studies to recruit larger, more diverse samples, and use statistical methods to examine comorbidity and different outcomes associated with sleep disturbance. The use of actigraphy has illuminated information about naturalistic sleep-wake patterns in BPD (e.g., [55•]), and technology enabling the use of actigraphy is becoming more affordable and efficient. The past decade has seen the discovery of new information about this relationship over the lifespan, perceptions of sleep in individuals with borderline pathology, attitudes towards sleep and medication, frequency and content of nightmares, and agreement between PSG findings and subjective beliefs about sleep quality. The combination of new and different methodologies that can be used for a more well-rounded assessment of sleep disturbance in BPD will answer new and exciting research questions about this relationship in the future.

Acknowledgments Preparation of this review was supported by a grant from the National Institute of Aging (RO1-AG045231). We would like to thank Merlyn Rodrigues and Betsy Edershile for their continued excellence on the St. Louis Personality and Aging Network research project.

\section{Compliance with Ethics Guidelines}

Conflict of Interest Joshua R. Oltmanns has received grants from the National Institute on Aging during the conduct of the study.

Thomas F. Oltmanns has received grants from the National Institute on Aging during the conduct of the study.

Human and Animal Rights and Informed Consent This article does not contain any studies with human or animal subjects performed by any of the authors.

\section{References}

Papers of particular interest, published recently, have been highlighted as:

- Of importance

1. American Psychiatric Association. Diagnostic and statistical manual of mental disorders. 5th ed. Washington, DC: Author; 2013.

2. Trull TJ, Jahng S, Tomko RL, Wood PK, Sher KJ. Revised NESA $\mathrm{RC}$ personality disorder diagnoses: gender, prevalence, and 
comorbidity with substance dependence disorders. J Personal Disord. 2010;24(4):412-26.

3. Baer RA, Peters JR, Eisenlohr-Mohl TA, Geiger PJ, Sauer SE. Emotion-related cognitive processes in borderline personality disorder: a review of the empirical literature. Clin Psychol Rev. 2012;32:359-69.

4. Bagge C, Nickell A, Stepp S, Durrett C, Jackson K, Trull TJ. Borderline personality disorder features predict negative outcomes 2 years later. J Abnorm Psychol. 2004;113:279-88.

5. Gunderson JG, Stout RL, McGlashan TH, Shea MT, Morey LC, Grilo CM, et al. Ten-year course of borderline personality disorder. Arch Gen Psychiatry. 2011;68:827-37.

6. Trull TJ, Sher KJ, Minks-Brown C, Durbin J, Burr R. Borderline personality disorder and substance use disorders: a review and integration. Clin Psychol Rev. 2000;20:235-53.

7. Duberstein PR, Conwell Y. Personality disorders and completed suicide: a methodological and conceptual review. Clin Psychol Sci Pract. 1997;4:359-76.

8. El-Gabalawy R, Katz L, Sareen J. Comorbidity and associated severity of borderline personality disorder and physical health conditions in a nationally representative sample. Psychosom Med. 2010;72:641-7.

9. Moran P, Stewart R, Brugha T, Bebbington P, Bhugra D, Jenkins R, et al. Personality disorder and cardiovascular disease: results from a national household survey. J Clin Psychiatry. 2007;68:69-74.

10. Powers AD, Oltmanns TF. Personality disorders and physical health: a longitudinal examination of physical functioning, healthcare utilization, and health-related behaviors in middle-aged adults. J Personal Disord. 2012;26:524-38.

11. Oltmanns JR, Weinstein Y, Oltmanns TF. Borderline personality pathology and insomnia symptoms in community-dwelling older adults. Personal Ment Health. 2014;8:178-87. An investigation of BPD and sleep in a large, representative sample of older adults in the community.

12. Schredl M, Paul F, Reinhard I, Ebner-Priemer UW, Schmahl C, Bohus M. Sleep and dreaming in patients with borderline personality disorder: a polysomnographic study. Psychiatry Res. 2012;200: 430-6. Another recent, thoughtful PSG investigation of BPD and sleep taking into account comorbidity with PTSD.

13. Morin CM, Jarrin DC. Epidemiology of insomnia: prevalence, course, risk factors, and public burden. Sleep Med Clin. 2013;281-297.

14. Talbot LS, Stone S, Gruber J, Hairston IS, Eidelman P, Harvey AG. A test of the bidirectional association between sleep and mood in bipolar disorder and insomnia. J Abnorm Psychol. 2012;121:3950 .

15. Gratz KL, Rosenthal MZ, Tull MT, Lejuez CW, Gunderson JG. Experimental investigation of emotion dysregulation in borderline personality disorder. J Abnorm Psychol. 2006;115:850-5.

16. American Psychiatric Association. Diagnostic and statistical manual of mental disorders. 3rd ed. Washington, DC: Author; 1980.

17. Bell J, Lycaki H, Jones D, Kelwala S, Sitaram N. Effect of preexisting borderline personality disorder on clinical and EEG sleep correlates of depression. Psychiatry Res. 1983;9:115-23.

18. Akiskal HS, Yerevanian BI, Davis GC, King D, Lemmi H. The nosologic status of borderline personality: clinical and polysomnographic study. Am J Psychiatry. 1985;142:192-8.

19. Battaglia M, Ferini-Strambi L, Smirne S, Bernardeschi L, Bellodi L. Ambulatory polysomnography of never-depressed borderline subjects: a high-risk approach to rapid eye movement latency. Biol Psychiatry. 1993;33:326-34.

20. Benson KL, King R, Gordon D, Silva JA. Sleep patterns in borderline personality disorder. J Affect Disord. 1990;18:267-73.

21. McNamara E, Reynolds III CF, Soloff PH, Mathias R, Rossi A, Spiker D, et al. EEG sleep evaluation of depression in borderline patients. Am J Psychiatry. 1984;141:182-6.
22. Benca RM, Okawa M, Uchiyama M, Ozaki S, Nakajima T, Shibui K, et al. Sleep and mood disorders. Sleep Med Rev. 1997;1:45-56.

23. Asaad T, Okasha T, Okasha T. Sleep EEG findings in CD-10 borderline personality disorder in Egypt. J Affect Disord. 2002;1(1-3): $11-8$.

24. De la Fuente JM, Bobes J, Morlan I, Bascaran MT, Vizuete C, Linkowski $\mathrm{P}$, et al. Is the biological nature of depressive symptoms in borderline patients without concomitant Axis I pathology idiosyncratic? Sleep EEG comparison with recurrent brief, major depression and control subjects. Psychiatr Res. 2004;129(1):65-73.

25. Hornung OP, Regen F, Warnstedt C, Anghelescu I, Danker-Hopfe $\mathrm{H}$, Heuser I, et al. Declarative and procedural memory consolidation during sleep in patients with borderline personality disorder. J Psychiatr Res. 2008;42:653-8.

26. Bastien CH, Guimond S, St-Jean G, Lemelin S. Signs of insomnia in borderline personality disorder individuals. J Clin Sleep Med. 2008;4:462-70. A recent and particularly well-done PSG examination of BPD patients in comparison to insomnia patients and normal controls.

27. De la Fuente JM, Bobes J, Vizuete C, Mendlewicz J. Borderline patients without concomitant major depression: a comparison with major depressives and normal control subjects. Psychiatry Res. 2001;105:87-95.

28. Reynolds III CF, Soloff PH, Kupfer DJ, Taska LS, Restifo K, Coble PA, et al. Depression in borderline patients: a prospective EEG sleep study. Psychiatry Res. 1985;14:1-15.

29. Lindberg N, Tani P, Appelberg B, Naukkarinen H, Rimon R, Porkka-Heiskanen T, et al. Human impulsive aggression: a sleep research perspective. J Psychiatr Res. 2003;37:313-24.

30. Philipsen A, Feige B, Al-Shajlawi A, Schmahl C, Bohus M, Richter $\mathrm{H}$, et al. Increased delta power and discrepancies in objective and subjective sleep measurements in borderline personality disorder. J Psychiatr Res. 2005;39:489-98.

31. American Academy of Sleep Medicine, Iber C. The AASM manual for the scoring of sleep and associated events: rules, terminology and technical specifications. American Academy of Sleep Medicine; 2007.

32. Buysse DJ, Reynolds III CF, Monk TH, Berman SR, Kupfer DJ. The Pittsburgh Sleep Quality Index: a new instrument for psychiatric practice and research. Psychiatry Res. 1989;28:193-213.

33. Semiz UB, Basoglu C, Ebrinc S, Cetin M. Nightmare disorder, dream anxiety, and subjective sleep quality in patients with borderline personality disorder. Psychiatry Clin Neurosci. 2008;62:48-55.

34. Simor P, Csoka S, Bodizs R. Nightmares and bad dreams in patients with borderline personality disorder: fantasy as a coping skill? Eur J Psychiatry. 2010;24:28-37.

35. Harty L, Forkner RD, Thompson A, Stuewig J, Tangney JP. Are inmates' subjective sleep problems associated with borderline personality, psychopathy, and antisocial personality independent of depression and substance dependence? J Forensic Psychiatry Psychol. 2010;21:23-38.

36. Morey LC. Professional manual for the Personality Assessment Inventory. Odessa: Psychological Assessment Resources; 1991.

37. Zanarini MC, Frankenburg FR, Hennen J, Reich DB, Silk KR. The McLean Study of Adult Development (MSAD): overview and implications of the first six years of prospective follow-up. J Personal Disord. 2005; 19:505-23.

38. Plante DT, Zanarini MC, Frankenburg FR, Fitzmaurice GM. Sedative-hypnotic use in patients with borderline personality disorder and axis II comparison subjects. J Personal Disord. 2009;23: 563-71.

39. Plante DT, Frankenburg FR, Fitzmaurice GM, Zanarini MC. Relationship between sleep disturbance and recovery in patients with borderline personality disorder. J Psychosom Res. 2013;74: 278-82. 
40. Plante DT, Frankenburg FR, Fitzmaurice GM, Zanarini MC. Relationship between maladaptive cognitions about sleep and recovery in patients with borderline personality disorder. Psychiatry Res. 2013;210:975-9.

41. Kessler RC, Merikangas KR. The National Comorbidity Survey Replication (NCS-R): background and aims. Int J Methods Psychiatr Res. 2004;13:60-8.

42. Selby EA. Chronic sleep disturbances and borderline personality disorder symptoms. J Consult Clin Psychol. 2013;81:941-7.

43. Oltmanns TF, Rodrigues MM, Weinstein Y, Gleason MEJ. Prevalence of personality disorders at midlife in a community sample: disorders and symptoms reflected in interview, self, and informant reports. J Psychopathol Behav Assess. 2014;36:177-88.

44. Lazarus SA, Cheavens JS, Festa F, Rosenthal MZ. Interpersonal functioning in borderline personality disorder: a systematic review of behavioral and laboratory-based assessments. Clin Psychol Rev. 2014;34:193-205.

45. Bastien $\mathrm{CH}$, Vallieres $\mathrm{A}$, Morin $\mathrm{CM}$. Validation of the Insomnia Severity Index as an outcome measure for insomnia research. Sleep Med. 2001;2:297-307.

46. Gunderson JG. Borderline personality disorder: ontogeny of a diagnosis. Am J Psychiatry. 2009;166:530-9.

47. Miller JD, Morse JQ, Nolf K, Stepp SD, Pilkonis PA. Can DSM-IV borderline personality disorder be diagnosed via dimensional personality traits? Implications for the DSM-5 personality disorder proposal. J Abnorm Psychol. 2012;121:944-50.
48. Widiger TA, Trull TJ. Plate tectonics in the classification of personality disorder: shifting to a dimensional model. Am Psychol. 2007;62:71-83.

49. Lynam DR, Widiger TA. Using the five-factor model to represent the DSM-IV personality disorders: an expert consensus approach. J Abnorm Psychol. 2001;3:401-12.

50. Hampson SE. Personality processes: mechanisms by which personality traits 'get outside the skin'. Annu Rev Psychol. 2012;63:31539.

51. Smith TW, Glazer K, Ruiz JM, Gallo LC. Hostility, anger, aggressiveness, and coronary heart disease: an interpersonal perspective on personality, emotion, and health. J Pers. 2004;72:1217-70.

52. Turiano NA, Spiro III A, Mroczek DK. Openness to experience and mortality in men: analysis of trait and facets. J Aging Health. 2012;24:654-72.

53. Mullins-Sweatt SN, Edmundson M, Sauer-Zavala S, Lynam DR, Miller JD, Widiger TA. Five-factor measure of borderline personality traits. J Pers Assess. 2012;94:475-87.

54. Battaglia M, Strambi LF, Bertella S, Bajo S, Bellodi L. First-cycle REM density in never-depressed subjects with borderline personality disorder. Biol Psychiatry. 1999;45:1056-8.

55. Bromundt V, Wirz-Justice A, Kyburz S, Opwis K, Dammann G, Cajochen C. Circadian sleep-wake cycles, well-being, and light therapy in borderline personality disorder. J Personal Disord. 2013;27:680-96. One of the first studies to use actigraphy to study circadian rhythms of BPD patients. 